\title{
Evolution of H-shaped dielectric resonator antenna for 5G applications
}

\author{
S. Z. N. Zool Ambia1, M. H. Jamaluddin², M. R. Kamarudin ${ }^{3}$, J. Nasir ${ }^{4}$, R. R. Selvaraju \\ ${ }^{1,24,5}$ Wireless Communication Centre (WCC), School of Electrical Engineering, Universiti Teknologi Malaysia (UTM), \\ Malaysia \\ ${ }^{3}$ Centre for Electronic Warfare, Information and Cyber, Cranfield Defence and Security, Cranfield University, UK
}

\begin{tabular}{l} 
Article Info \\
\hline Article history: \\
Received Sep 24, 2018 \\
Revised Nov 25, 2018 \\
Accepted Dec 6, 2018 \\
\hline Keywords: \\
5G \\
Dielectric resonator antenna \\
(DRA) \\
Microstrip slot aperture (MSA) \\
Millimeter wave
\end{tabular}

\begin{abstract}
In this paper, an H-shaped Dielectric Resonator Antenna (DRA) with a Microstrip Slot Aperture (MSA) is presented and investigated at $26 \mathrm{GHz}$. In order to widen the bandwidth operation, the slot aperture feeding technique is applied. The designed DRA with relative permittivity, er of 10 is mounted on a Duroid substrate with a relative permittivity, er of 2.2, loss tangent of 0.0009 and a thickness of $0.254 \mathrm{~mm}$. The proposed antenna with overall size of $20 \times 20 \times 5.27 \mathrm{~mm} 3$ achieves good impedance matching, gain of $7.61 \mathrm{~dB}$ and good radiation patterns. An impedance bandwidth of $21.44 \%$, covering the frequency range from $24.72 \mathrm{GHz}$ to $30.62 \mathrm{GHz}$ made the antenna has potential for millimeter wave and $5 \mathrm{G}$ applications.
\end{abstract}

Copyright $@ 2019$ Institute of Advanced Engineering and Science. All rights reserved.

\section{Corresponding Author:}

Mohd Haizal Jamaluddin, Wireles Communication Centre, School of Electrical Engineering,

Universiti Teknologi Malaysia,

81310 Skudai, Johor Malaysia.

Email: haizal@fke.utm.my

\section{INTRODUCTION}

Wireless communication has become a very popular medium to everyone in the world after the complete wireless system has been launched in the year of 1897. This no wired communication has replaced the older version of communication method such as telegram. The process of sending and receiving the information can be carried out even if the sender and receiver did not facing each other. The first generation, $1 \mathrm{G}$ used analog system and about ten years after that, second generation known as $2 \mathrm{G}$ take in place. The system keep growing rapidly to third generation (3G) and fourth generation (4G) which every developments offers better feature to the users. Now, it is time for fifth generation $(5 \mathrm{G})$ to become reality and users can enjoy the benefits and improved features offered.

Along with the development of the communication system, the growth of sophisticated electrical and electronics devices in this advanced world has given a hard time for communication field to fulfill the mankind need. Almost all devices in communication systems use current cellular spectrum, which is below 3 $\mathrm{GHz}$ which makes this frequency spectrum becomes congested [1]-[4]. Considering this problem, an alternative way to avoid the overcrowded frequency spectrum is to utilize the less used frequency spectrum which is at millimeter wave bands at 30-300 GHz [5]. The devices need 5G cellular network to be operated at this bands.

The frequency of millimeter wave bands is very high. Thus, the size of the antenna needs to be very small. A small size of antennae is complicated to be fabricated and analyzed. Therefore, Dielectric Resonator Antenna (DRA) is the right choice to be used as the antenna material compared to the conventional antenna such as microstrip patch antenna. DRA has become research interest in the last two decades due to its very 
small size, light weight, easy excitation and large impedance bandwidth [6], [7]. DRA also offers low loss as the material is not made from metal [8]. Microstrip patch antenna is not a suitable choice to be implemented in the design as the antenna will suffer of high conductor losses at high frequency [9], [10].

Without modification, basic DRA such as rectangular, cylindrical or hemispherical shape will not boost the bandwidth to the higher value. In [11], the bandwidth obtained by the rectangular-shaped antenna is only about $2.1 \%$. While in [12], the cylindrical-shaped antenna produce a narrow bandwidth. The impedance bandwidth achieved after the simulation is only $4.8 \%$. So, the concern of the design is to increase the bandwidth as the main aspects in current DRA research design also focusing on this matter.

Theoretically, the wavelength of the antenna is inversely proportional to the frequency. An antenna designed at millimeter wave requires higher frequency to ensure a smaller wavelength of the antenna obtained. Therefore, due to one of the advantages of the DRA which it can be designed in a very small size, while giving wide impedance bandwidth, an H-shaped DRA has been proposed in this paper. The antenna is designed at higher frequency, $26 \mathrm{GHz}$ which is suitable to be used for 5G applications. The evolution of the final design of the antenna is simulated and presented.

\section{ANTENNA DESIGN}

The initial dimensions of DRA are determined using the equations developed for the magnetic wall waveguide model in the transverse plane $(\mathrm{x}-\mathrm{z})$ by considering $\mathrm{m}=\mathrm{n}=1$. By matching the fields at the boundary conditions, the wave propagation numbers can be determined by the following equations:

$$
\begin{aligned}
& k_{x}=\frac{m \pi}{a} \\
& k_{y}=\frac{n \pi}{b} \\
& k_{x}^{2}+k_{y}^{2}+k_{z}^{2}=\varepsilon_{r} k_{o}^{2}
\end{aligned}
$$

where $\mathrm{kx}, \mathrm{ky}$, and $\mathrm{kz}$ denote the wave-numbers along $\mathrm{x}, \mathrm{y}$, and $\mathrm{z}$ directions, respectively, inside the DR also should satisfy:

$$
k_{z} \tan \left(k_{z} d / 2\right)=\sqrt{(\varepsilon r-1) k o^{2}-k z^{2}}
$$

where ko denotes the wave-number in the free space.

Figure 1 shows the transition from Rectangular Dielectric Resonator Antenna (RDRA) to the proposed H-shaped DRA in this paper. RDRA with the dimension of 3.5 X $3.5 \mathrm{X} 4.94 \mathrm{~mm}$ is designed at 26 GHz. In order to increase the bandwidth, the RDRA is modified to another shape, which is U-shaped DRA. A small rectangle is cut out at the top part of the DR to make a U-shaped antenna.



Figure 1. The transition of H-shaped DRA

The purpose of modification of the DRA is to decrease the $\mathrm{Q}$ factor of the resonator. The relationship of the $\mathrm{Q}$ factor and bandwidth is inversely proportional. The decreasing value of $\mathrm{Q}$ factor will results in increasing value of bandwidth. This condition obeys the following equation:

$$
B W=\frac{S-1}{Q \sqrt{S}}
$$

$$
\begin{aligned}
& \text { where: } \mathrm{BW}=\text { Bandwidth } \\
& \mathrm{S} \quad=\text { Maximum acceptable VSWR } \\
& \text { Q = Q-factor }
\end{aligned}
$$


Lastly, the modification ends with H-shaped DRA. The same dimension of the small rectangle at the top part of the DR is used at another side of the DR to realize an H-shaped DRA. The size of the small rectangle is varied to get the optimum dimension to fulfill the design requirement.

The geometry of the designed antenna after two modifications is shown in Figure 2. The material used for Dielectric Resonator (DR) is ECCOSTOCK@HiK having relative permittivity of 10 and tangent constant of 0.002 . The DR is mounted on a $20 \mathrm{X} 20 \mathrm{~mm} 2$ Duroid substrate which has relative permittivity, er of 2.2, loss tangent of 0.0009 and a thickness of $0.254 \mathrm{~mm}$.

The DR is fed by microstrip slot aperture. Microstrip slot aperture feeding technique is chosen considering the fulfillment of the $5 \mathrm{G}$ requirements which is to have high directivity antenna and high gain. The uses of microstrip slot aperture technique are also to provide wide bandwidth operation. Another advantage of slot aperture is the spurious radiation can be avoided since the feed network is located below the ground plane. After several analysis and simulation made using CST Microwave Studio 2015 software, the optimum DRA dimensions is obtained. The dimensions of the DRA are as follows: $\mathrm{L} 1=\mathrm{W} 1=3.5 \mathrm{~mm}$, $\mathrm{L} 2=0.45 \mathrm{~mm}, \mathrm{~W} 2=0.40 \mathrm{~mm}$ and $\mathrm{h}=4.94 \mathrm{~mm}$.

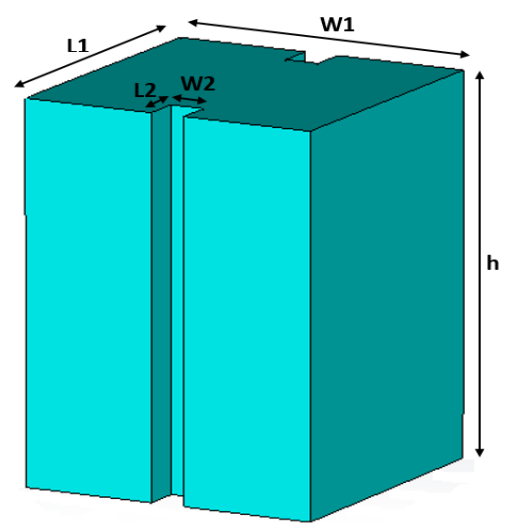

Figure 2. The optimum dimension of the proposed antenna

\section{RESULTS AND DISCUSSIONS}

The CST Microwave Studio 2015 software is used to optimize the antenna and to analyze the parametric analysis. The parametric analysis of the Dielectric Resonator Antenna (DRA) by varying the size of the rectangle that is a part of the DR that has been cut out to get the optimum dimension is presented. Figure 3 shows the simulation result for reflection coefficient of $\mathrm{H}$-shaped antenna after varying three sizes of the rectangle DR that has been cut out.

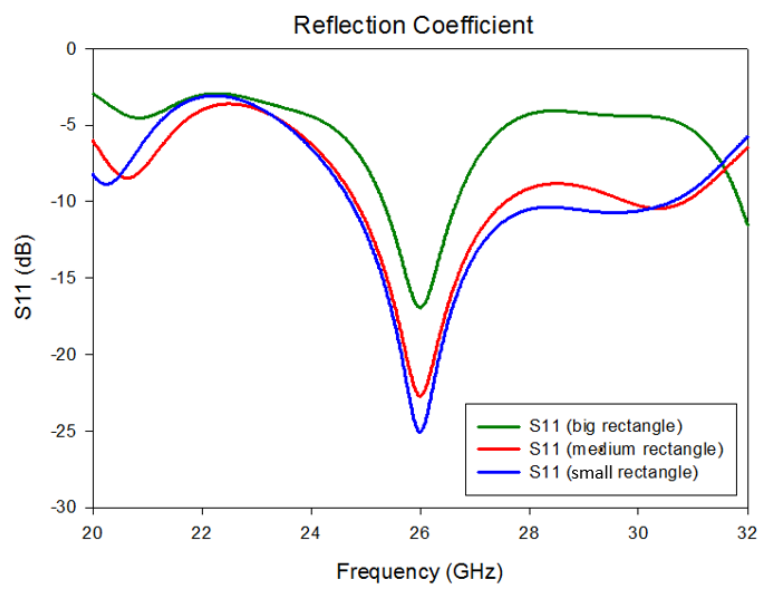

Figure 3. The reflection coefficient of three designs of H-shaped DRA 
The purpose of varying the rectangle DR is to obtain the optimum size in order to get the widest bandwidth possible. The elimination of the rectangle DR is started with a big dimension which is $1 \mathrm{X} 1.25$ $\mathrm{mm}$, followed by medium size of $0.7 \mathrm{X} 0.85 \mathrm{~mm}$ and lastly, $0.4 \mathrm{X} 0.45 \mathrm{~mm}$. The bandwidth off all three simulations has been recorded in Table 1 . From Table 1, it is clearly stated that the smallest cut gives the highest bandwidth, $5.9 \mathrm{GHz}$ which is equivalent to $21.44 \%$. The smallest cut DR also has benefit in thickness of the DR, where the thickness is the lowest compared to the others. So, the overall size of the antenna can be reduced.

\begin{tabular}{|c|c|c|c|}
\hline Design & $\begin{array}{c}\text { Frequency } \\
\text { Range } \\
(\mathrm{GHz})\end{array}$ & $\begin{array}{c}\text { Bandwidth } \\
\text { (GHz) } \\
(\%)\end{array}$ & $\begin{array}{c}\text { DR } \\
\text { Thickness } \\
\text { (mm) }\end{array}$ \\
\hline $\begin{array}{l}1 \mathrm{X} 1.25 \mathrm{~mm} \\
\text { (big) }\end{array}$ & $25.322-26.674$ & $\begin{array}{c}1.352 \\
(5.20 \%)\end{array}$ & 6.70 \\
\hline $\begin{array}{l}0.7 \times 0.85 \mathrm{~mm} \\
\quad \text { (medium) }\end{array}$ & $24.820-27.581$ & $\begin{array}{c}2.761 \\
(10.55 \%)\end{array}$ & 5.28 \\
\hline $\begin{array}{c}0.4 \times 0.45 \mathrm{~mm} \\
(\mathrm{small})\end{array}$ & $24.722-30.621$ & $\begin{array}{c}5.899 \\
(21.44 \%)\end{array}$ & 4.94 \\
\hline
\end{tabular}

Table 2 presents the result recorded for the evolution of the antenna from rectangular to H-shape DRA, containing frequency range, bandwidth and gain of the evolution process. Bandwidth of $17.43 \%$ is obtained when no part of the DR is cut out. The first elimination as shown in Figure 4 results in bandwidth increment from $17.43 \%$ to $19.72 \%$. From U-shaped DRA, the process proceeds with the second elimination to form $\mathrm{H}$-shaped DRA as illustrated in Figure 5. After simulation made, the bandwidth again gives a positive result when it increase to $21.44 \%$. Beside the increment of the bandwidth, gain obtained from the design is also increased along with the evolution process. The highest gain this antenna can reach in this paper is $7.61 \mathrm{~dB}$.

\begin{tabular}{|c|c|c|c|}
\hline Pesign & $\begin{array}{c}\text { Frequency } \\
\text { Range } \\
(\mathrm{GHz})\end{array}$ & $\begin{array}{c}\text { Bandwidth } \\
(\mathrm{GHz}) \\
(\%)\end{array}$ & $\begin{array}{l}\text { Gain } \\
\text { (dB) }\end{array}$ \\
\hline Rectangular & $24.749-29.456$ & $\begin{array}{c}4.707 \\
(17.43 \%)\end{array}$ & 7.56 \\
\hline U-shape & $24.745-30.129$ & $\begin{array}{c}5.384 \\
(19.72 \%)\end{array}$ & 7.59 \\
\hline H-shape & $24.722-30.621$ & $\begin{array}{c}5.899 \\
(21.44 \%)\end{array}$ & 7.61 \\
\hline
\end{tabular}



Figure 4. First elimination of rectangle part of the DRA

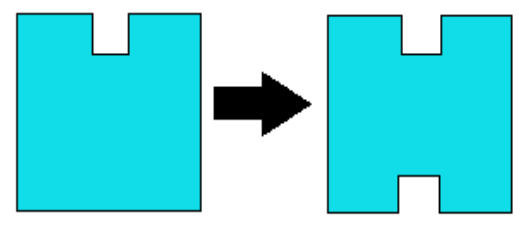

U-shaped DRA H-shaped DRA

Figure 5. Second elimination of rectangle part of the DRA

The far-field radiation pattern is also simulated and recorded. The radiation pattern is simulated in the $\mathrm{x}-\mathrm{z}$ plane (E-plane) and $\mathrm{y}-\mathrm{z}$ plane (H-plane). Both E-plane (Figure 6) and H-plane (Figure 7) radiation pattern has broadside direction. The gain of the antenna which is $7.61 \mathrm{~dB}$ is shown along with the 3D radiation pattern plot as in Figure 8. 
Farfield Realized Gain Abs (Phi=90)

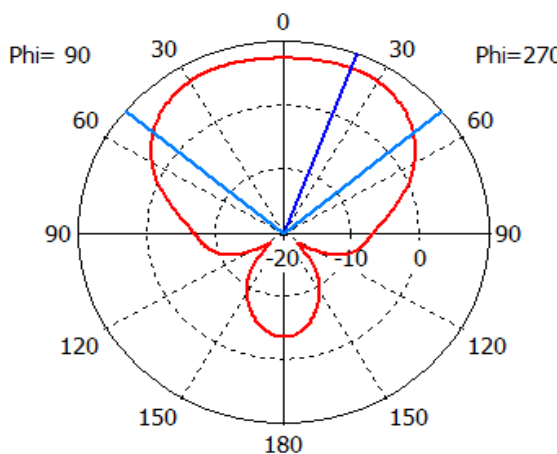

Theta / Degree vs. dB
Farfield Realized Gain Abs (Phi=0)

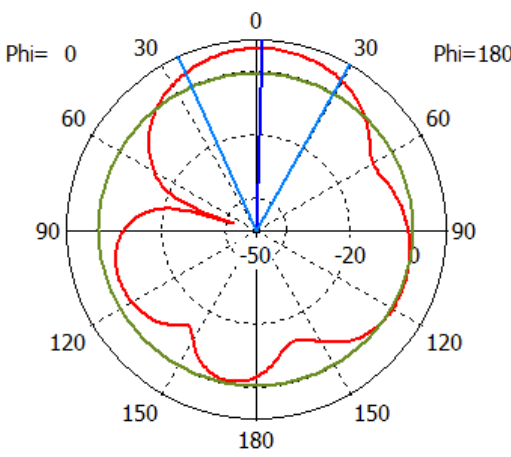

Theta / Degree vs. dB

Figure 6. E-plane radiation pattern

Figure 7. H-plane radiation pattern

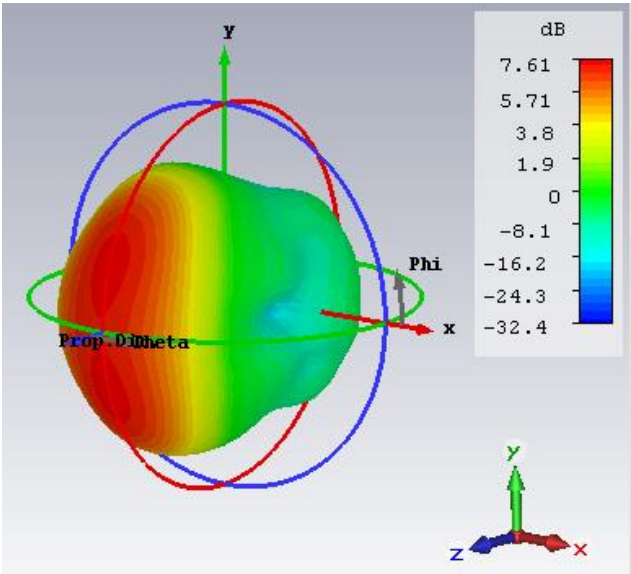

Figure 8. Far field plot in 3D

\section{CONCLUSION}

The evolution of H-shaped Dielectric Resonator Antenna (DRA) for 5G Applications is presented. The design of H-shaped DRA is completed after two modifications made, firstly the rectangular DRA is modified to U-shaped DRA and secondly to H-shaped DRA. Slot aperture feeding technique and the modification of the antenna is used to obtain large bandwidth operation. The frequency range of the antenna is between $24.72 \mathrm{GHz}$ to $30.62 \mathrm{GHz}$ and the resonance frequency is $26 \mathrm{GHz}$. The bandwidth of $21.44 \%$ is achieved and the reflection coefficient, S11 $<-10 \mathrm{~dB}$ of -25.10 is obtained. Good radiation pattern and gain of $7.61 \mathrm{~dB}$ are presented. Previous researches of the unmodified rectangular DRA have only $2.1 \%$ bandwidth and $4.8 \%$ for cylindrical DRA. Bandwidth of the proposed antenna is about ten times larger than previous research of rectangular DRA and five times bigger than cylindrical DRA. The increment of the bandwidth is obtained by a slight modification of the DRA geometry. Many of the previous researches focus on the lower frequency. As the lower frequencies are congested from various communication systems, this proposed antenna is designed at higher frequency to avoid the crowded and to obtain wider bandwidth. The overall size of the antenna is very small which is only $20 \times 20 \times 5.27 \mathrm{~mm} 3$ and the antenna design is attractive. From the results demonstrated by the design, this H-shaped DRA can be a potential antenna to be used in millimeter wave and $5 \mathrm{G}$ applications.

\section{ACKNOWLEDGEMENTS}

The authors from Universiti Teknologi Malaysia would like to thank the Ministry of Education (MOE) and UTM GUP (votes 13H26), UTM Flagship (03G59), FRGS (4F733) and HiCoE Grant (Votes 4J220) for sponsoring this work. 


\section{REFERENCES}

[1] Z. Pi, F. Khan, and E. Samsung, “An Introduction to Millimeter-Wave Mobile Broadband Systems,"IEEE Communications Magazine, vol. 49, pp. 101-107, 2011.

[2] T. S. Rappaport, et al., "Millimeter-Wave Mobile Communications for 5G cellular: It Will Work!,"IEEE Access,vol. 1, pp. 335-349, 2013.

[3] L. Wei, et al., "Key Elements to Enable Millimeter Wave Communications for 5G Wireless Systems," IEEE Wireless Communications, vol. 21, pp. 136-143, 2014.

[4] N. M. Nor, M. H. Jamaluddin, "A Dual Band Mimo Dielectric Resonator Antenna for WLAN Application”, Jurnal Teknologi (Sciences \& Engineering), vol. 77, pp. 1-4, 2015.

[5] Wells. "Faster than fiber: The future of Multi-Gb/s Wireless", IEEE Microwave Magazine, vol.10, no.3, pp.104112, 2009.

[6] Y. Ding, K. W. Leung, "On the Dual-Band DRA-Slot Hybrid Antenna," IEEE Transaction on Antennas and Propagation, vol. 57, pp. 624-630, 2009.

[7] J. Nasir, et al., "A Reduced Size Dual Port MIMO DRA with High Isolation for 4G Application," International Journal of RF and Microwave Computer-Aided Engineering, Vol. 14(6), pp. 495- 501, 2015.

[8] Y. He, et al., "The Design of A Tripolarization Rectangle Dielectrcic Resonator Antenna," 2016 10th European Conference on Antennas and Propagation (EuCAP), pp. 1-3, 2016.

[9] R. Chair, A. A. Kishk, and K. F. Lee, "Widenband stair-shaped dielectric resonator antennas," IEEE Microwave Antennas Propagation, vol. 1, no. 2, pp. 299-305, 2007.

[10] M Abedian, et al., "Novel Design of Compact UWB Dielectric Resonator Antenna With Dual-Band-Rejection Characteristics for WiMAX/WLAN Bands", IEEE Antenna and Wireless Propagation Letters., vol. 14, pp. 245248, 2015.

[11] P. R. Pimpalgaonkar, et al. "Design of Rectangular and Hemispherical Dielectric Resonator Antenna," 2016 International Conference on Communication and Signal Processing (ICCSP), pp. 1430-1433, 2016.

[12] S A. Panigrahi and S. K. Behera, "H-shaped Slot Coupled Dual-Polarized Dielectric Resonator Antenna for C-band Applications." 2015 Global Conference on Communication Technologies (GCCT), pp. 719-722, 2015.

\section{BIOGRAPHIES OF AUTHORS}

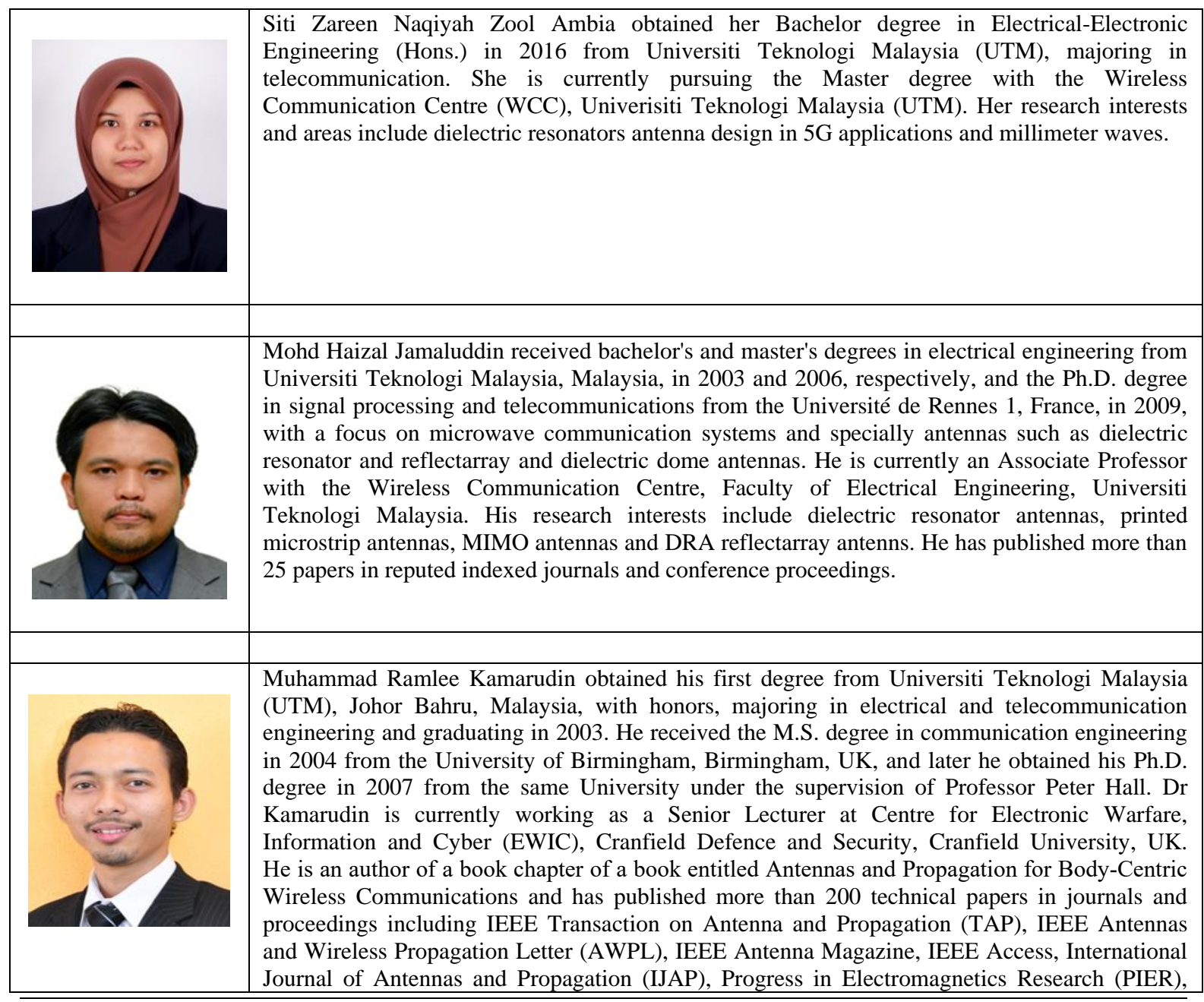

Evolution of H-shaped dielectric resonator antenna for $5 G$ applications (S. Z. N. Zool Ambia) 


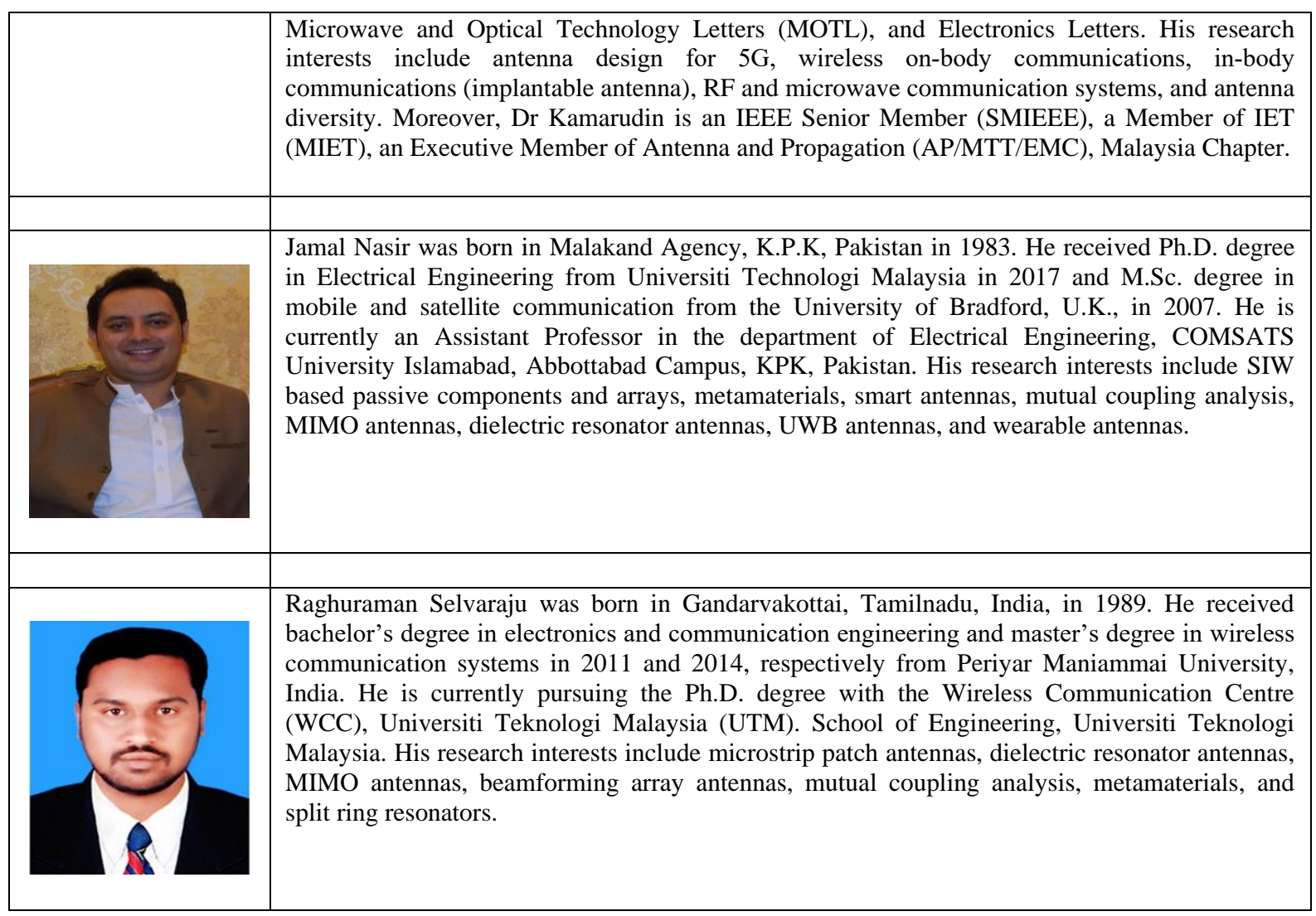

